\title{
Differential expression of mTOR components in endometriosis and ovarian cancer: Effects of rapalogues and dual kinase inhibitors on mTORC1 and mTORC2 stoichiometry
}

\author{
KARLY-RAI ROGERS-BROADWAY ${ }^{1}$, JUHI KUMAR ${ }^{1}$, CRISTINA SISU $^{1}$, GURLEEN WANDER $^{2}$, EMILY MAZEY $^{1}$, \\ JEYAROOBAN JEYANEETHI $^{1}$, GEORGE PADOS ${ }^{3}$, DIMITRIS TSOLAKIDIS ${ }^{3}$, ELEFTHERIOS KLONOS ${ }^{3}$, \\ THOMAS GRUNT $^{4}$, MARCIA HALL ${ }^{1,5}$, JAYANTA CHATTERJEE ${ }^{6 *}$ and EMMANOUIL KARTERIS ${ }^{1 *}$ \\ ${ }^{1}$ Division of Biosciences, College of Health and Life Sciences, Brunel University London, Uxbridge UB8 3PH; \\ ${ }^{2}$ Queen Charlotte and Chelsea Hospital, London W120HS, UK; ${ }^{3}$ First Department of OB-GYN, \\ 'Papageorgiou' Hospital, University of Thessaloniki Medical School, Thessaloniki 54124, Greece; \\ ${ }^{4}$ Ludwig Boltzmann Cluster Oncology and Division of Oncology, Department of Medicine I, \\ Medical University Vienna, A-1090 Vienna, Austria; ${ }^{5}$ Mt Vernon Cancer Centre, Harefield HA6 2RN; \\ ${ }^{6}$ Faculty of Health and Medical Sciences, School of Biosciences and Medicine, \\ University of Surrey, Guildford, Surrey GU2 7XH, UK
}

Received March 26, 2018; Accepted September 4, 2018

DOI: $10.3892 /$ ijmm.2018.3967

\begin{abstract}
Endometriosis is a well-known risk factor for ovarian cancer. The genetic changes that characterise endometriosis are poorly understood; however, the mechanistic target of rapamycin (mTOR) pathway is involved. In this study, we investigated the expression of key mTOR components in endometriosis and the effects of rapalogues using an endometrioid ovarian carcinoma cell line (MDAH 2774) as an in vitro model. Gene expression of mTOR, DEPTOR, Rictor and Raptor was assessed by qPCR in 24 endometriosis patients and in silico in ovarian cancer patients. Furthermore, the effects of Rapamycin, Everolimus, Deforolimus, Temsirolimus, Resveratrol, and BEZ235 (Dactolisib, a dual kinase inhibitor) on mTOR signalling components was assessed. mTOR showed a significant increase in the expression in endometriosis and ovarian endometrioid adenocarcinoma patients compared to non-affected controls. DEPTOR, an inhibitor of mTOR, was downregulated in the advanced stages of ovarian cancer (III and IV) compared to earlier stages (I and II). Treatment of MDAH-2774 cells with the mTOR inhibitors resulted in the significant upregulation of DEPTOR mRNA, whereas treatment with rapamycin and BEZ-235 $(100 \mathrm{nM})$ resulted in downregulation of the mTOR protein expression after $48 \mathrm{~h}$ of treatment. None of the treat-
\end{abstract}

Correspondence to: Dr Emmanouil Karteris, Division of Biosciences, College of Health and Life Sciences, Brunel University London, Kingston Lane, Uxbridge UB8 3PH, UK

E-mail: emmanouil.karteris@brunel.ac.uk

"Contributed equally

Key words: ovarian cancer, mTOR, rapalogues, endometriosis ments resulted in translocation of mTOR from cytoplasm to nucleus. Upregulation of DEPTOR is a positive prognostic marker in ovarian cancer and is increased in response to mTOR pathway inhibition suggesting that it functions as a tumour suppressor gene in endometrioid ovarian carcinoma. Collectively, our data suggest the mTOR pathway as a potential connection between endometriosis and ovarian cancer and may be a potential target in the treatment of both conditions.

\section{Introduction}

Endometriosis is a non-malignant condition characterised by the ectopic implantation and growth of endometrial tissue in locations within the abdominal cavity, such as the fallopian tubes, ovaries, peritoneum, vagina, bladder, bowel and rectum. Ectopic endometrium responds to hormonal changes in the same way as eutopic endometrium, by proliferating and shedding with the menstrual cycle causing pain and inflammation in the affected areas. Symptoms of endometriosis include dysmenorrhea, dyspareunia, dysuria, dyschezia, infertility and chronic pelvic pain as well as an increased risk of allergies, asthma, fibromyalgia, autoimmune disease, hypothyroidism, multiple sclerosis and chronic fatigue syndrome $(1,2)$. Endometriosis is estimated to affect around two million individuals in the UK (2).

Endometriosis is now becoming a well-documented risk factor for ovarian cancer, occurring in up to $28 \%$ of ovarian cancer patients (3-13). A 20-year study by Stewart et al, of more than 21,000 patients seeking fertility treatment in Western Australia has shown that endometriosis is particularly associated with an increased rate of ovarian cancer in patients who remained childless after fertility treatment (4). The study did not classify invasive epithelial ovarian cancer any further; however, a similarly large pooled analysis of 13 ovarian cancer risk factor studies examined the relationship between endome- 
triosis and the four major subtypes of epithelial ovarian cancer: serous (high and low grade), clear cell, endometrioid and mucinous (4). They found that the association between endometriosis and ovarian cancer differed between subtypes. Patients with low-grade serous (9.2\%), endometrioid (13.9\%) and clear cell $(20.2 \%)$ subtypes were more likely to have endometriosis (compared to $6.2 \%$ of control cases). Mucinous epithelial ovarian cancer was not at all associated with endometriosis (5). In the aforementioned studies, there was no increased risk of ovarian cancer in patients with pelvic inflammatory disorder, ovarian cysts, fibroids, breastfeeding, weight, height, body-mass index and tubal ligation, highlighting the unique relationship between endometriosis and ovarian cancer $(4,5,9)$.

The mTOR pathway is a central regulator of growth, proliferation, apoptosis and angiogenesis providing balance between cellular resources such as amino acids and growth factors and stresses such as hypoxia to control cellular behaviour accordingly. The activities of this pathway are mediated through the mTOR complex 1 (mTORC1) and mTOR complex 2 (mTORC2). mTORC1 contains mTOR, Raptor, DEPTOR, GBL and PRAS 40. mTORC2 contains mTOR, Rictor, DEPTOR and GBL. mTOR is a serine/threonine kinase and is the central catalytic component of mTORC1 and mTORC2. DEPTOR is a component that inhibits the activities of both mTORC1 and mTORC2 by direct interaction with mTOR. Raptor is a protein component of mTORC1 only and controls mTOR kinase activity. Rictor is a component of mTORC2 only. The pathways, including upstream regulators and downstream effectors of mTORC 1 and mTORC2 are shown in Fig. 1, in an abbreviated manner. The mTOR pathway is inhibited via Rapamycin, also known as sirolimus, which was first described in 1975. Since its discovery, a host of semi-synthetic Rapamycin-related mTORC1 inhibitors known as rapalogues, including Everolimus, Deforolimus and Temsirolimus have been developed. Besides these, dual mTOR and PI3K inhibitors such as NVP-BEZ235 and Resveratrol have also gained attention (14-16).

The genetic changes that characterise endometriosis are poorly understood; however, the mTOR pathway is involved in several ways. Leconte et al, showed an increased expression of Akt and p70S6K in both the eutopic endometrium and endometriotic lesion of patients with deep infiltrating endometriosis (DIE) in comparison to the control endometrium (17). Afshar et al, showed changes in 23 PI3K/Akt pathway-related genes and 26 MAP kinase pathway-related genes in the eutopic endometrium of baboons with endometriosis (18). Both the PI3K and MAP kinase pathways can inactivate the TSC1/TSC2 complex causing a release of its inhibitory effect on mTOR kinase activity (19-21). Guo et al, identified higher phosphorylated mTOR in ectopic endometrium than in eutopic or control endometria (22).

Due to its involvement in growth factor and energy sensing and its effect on protein synthesis and cell cycle progression, the mTOR pathway is involved in a range of malignancies. Although there is evidence for the involvement of the mTOR pathway in endometriosis and ovarian cancer, its role in the transformation from one condition to the other is poorly understood. In the present study, we examined the expression of key mTOR components in endometriosis and ovarian cancer and used an ovarian cancer cell line of endometrioid origin as a preclinical model to dissect further the mechanisms of action of mTOR inhibitors.

\section{Materials and methods}

Clinical samples. Tissue samples from patients with endometriosis $(n=24)$, and non-affected controls $(n=34)$ were obtained at the First Department of Obstetrics and Gynaecology, 'Papageorgiou' Hospital, University of Thessaloniki, Thessaloniki, Greece. Ethical approval was obtained by the Ethics Committee of Papageorgiou Hospital. For the circulating tumour cell (CTC) study, ovarian cancer blood samples $(n=3)$ were collected from ovarian cancer patients enrolled on a clinical trial at Mount Vernon Hospital, London, UK, known as CICATRIx. The present study was also approved by the National Research Ethics Committee and the Research and Development department of East and North Hertfordshire NHS Trust (26/7/16-present).

Written informed consent was obtained from all the patients. Patients were stage III/IV ovarian serous adenocarcinoma, and enrolled on the trial to begin third-line dual chemotherapy treatment. We confirm that all participants of this study from both clinical settings have given their consent regarding inclusion of material pertaining to themselves, as specified in the Declaration of Helsinki. None of the patients and controls can be identified via this manuscript as all cohorts are fully anonymized.

RNA isolation, cDNA synthesis and quantitative RT-PCR. RNA was extracted from tissue lysate using the GenElute ${ }^{\mathrm{TM}}$ mRNA MiniPrep kit (Sigma-Aldrich; Merck KGaA, Darmstadt, Germany). cDNA was synthesised from mRNA using Superscript II (Invitrogen; Thermo Fisher Scientific, Massachusetts, MN, USA). cDNA concentration was normalised using RNA concentrations determined by NanoDrop (Thermo Fisher Scientific, Pittsburgh, PA, USA). Relative expression of the genes of interest was assessed by quantitative PCR (qPCR) on an xxpress ${ }^{\circledR}$ (BJS Biotechnologies, Middlesex, UK) using Kapa SYBR Fast Universal Master Mix (KapaBiosystems-Sigma-Aldrich; Merck KGaA). Primers for mTOR, DEPTOR, Rictor and Raptor and qRT-PCR quantification were used as previously described (23). The following primers were used as housekeeping genes: $Y W H A Z$, forward: 5'-agacggaaggtgctgagaaa-3', and reverse: 5'-gaagcattggggatc aagaa-3'; and RPL13A, forward: 5'-cctggtctgagcccaataaa-3' and reverse: 5'-cttgctcccagcttcctatg-3'.

Tissue microarray. Paraffin-embedded ovarian tissue microarray slides each containing 70 clinical samples were purchased from US Biomax (Rockville, MD, USA). The slides were deparaffinised and rehydrated, followed by antigen retrieval. Blocking was carried out with $5 \%$ goat serum, followed by 24 -h incubation with primary rabbit monoclonal antibodies for mTOR (catalog no. 2983, Cell Signalling Technology, Inc., Danvers, MA, USA; dilution 1:200), and DEPTOR (catalog no. 11816, Cell Signalling Technology, Inc.; dilution 1:200). Following numerous washes with PBS, the slides were incubated with HRP-conjugated secondary antibody for $60 \mathrm{~min}$. The slides were then washed and subjected to DAB staining, counterstained with haematoxylin and washed with $0.1 \%$ sodium bicarbonate. The slides were then analysed for immunoreactivity of both proteins by light microscope (Zeiss, Jena, Germany) and positive results were measured by the percentage of positive tumour cells. 


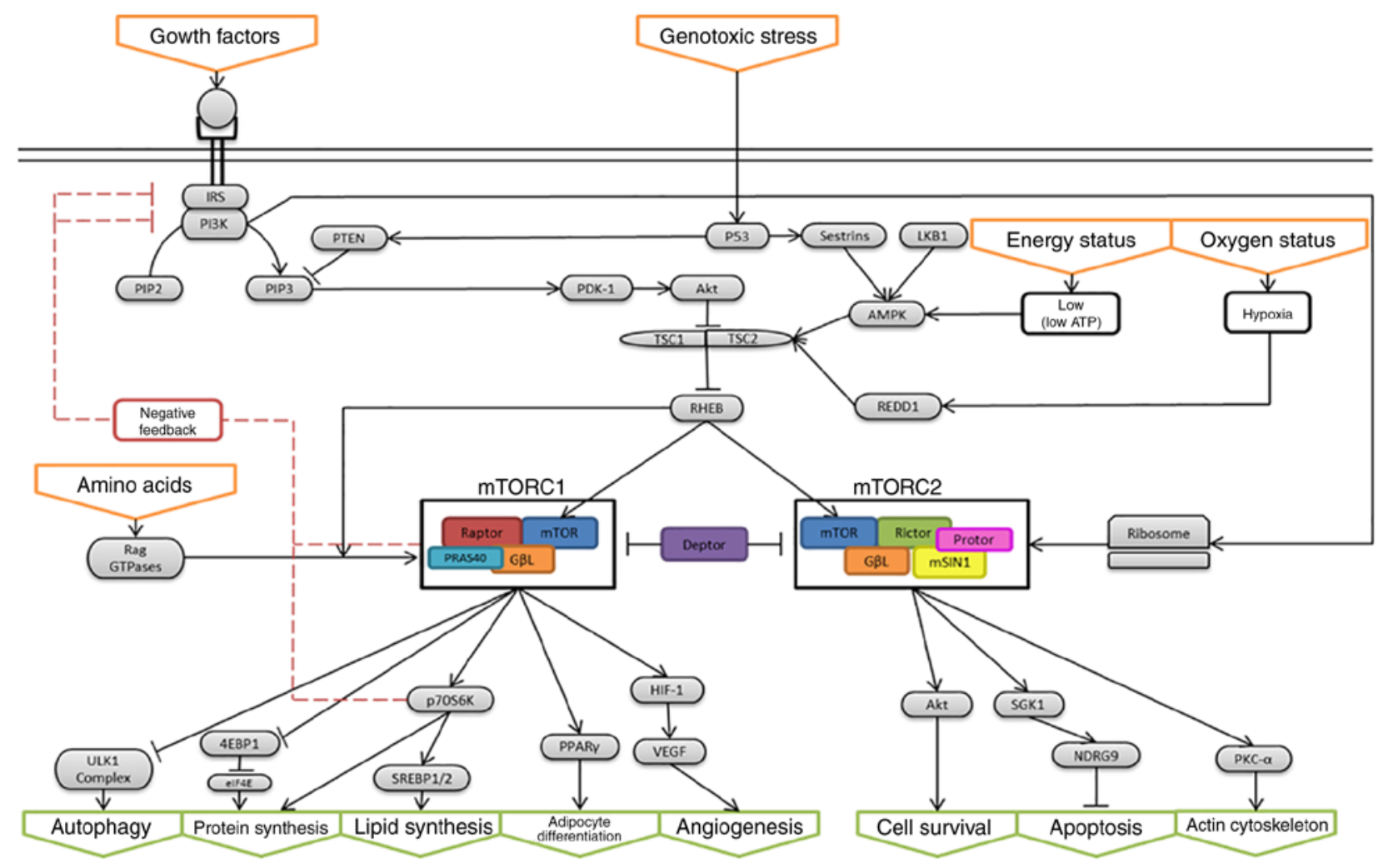

Figure 1. An overview of mTOR signalling, including upstream and downstream regulators.

Cell line and treatments. MDAH-2774 cells were purchased from ATCC and are human ovarian endometrioid adenocarcinoma-derived. MDAH-2774 cells were grown in DMEM (Dulbecco's Modified Eagle's medium; Life Technologies; Thermo Fisher Scientific) supplemented with 10\% FBS (Life Technologies; Thermo Fisher Scientific), $1 \%$ penicillin/streptomycin (Life Technologies; Thermo Fisher Scientific) and 1\% L-glutamine (Life Technologies; Thermo Fisher Scientific). MDAH-2774 cells were cultured at $37^{\circ} \mathrm{C}$ at $5 \% \mathrm{CO}_{2}$. We studied the effect of mTOR pathway inhibition on the gene expression of mTOR, Rictor, DEPTOR, Raptor in vitro using six inhibitory agents: Rapamycin (Sigma-Aldrich; Merck KGaA), Everolimus (Sigma-Aldrich; Merck KGaA), Deforolimus (Selleckchem, Houston, TX, USA), Temsirolimus (Sigma-Aldrich; Merck KGaA), NVP-BEZ235 (Selleckchem) and resveratrol (Sigma-Aldrich; Merck KGaA). Treatments were applied in varying concentrations as given in Table I for 24,48 and $72 \mathrm{~h}$.

Immunofluorescence. Following treatments, MDAH-2774 cells were fixed for $10 \mathrm{~min}$ in $4 \%$ paraformaldehyde, followed by incubation with $10 \%$ bovine serum albumin (BSA) for $1 \mathrm{~h}$. MDAH-2774 cells were incubated overnight at $4^{\circ} \mathrm{C}$ with mTOR primary rabbit monoclonal antibody (catalog no. 2983, Cell Signalling Technology, Inc.), followed by the addition of a secondary anti-rabbit Alexa Fluor ${ }^{\circledR}$ 488) (Thermo Fisher Scientific, Pittsburgh, PA, USA; dilution 1:400) and visualisation as previously described (20). ImageJ software was used to analyse the staining intensity. For each image, 10 individual cells were captured using the freehand tool, ensuring that the area for each cell was kept approximately the same. A back-
Table I. Details of the mTOR pathway inhibitory agents used in this study. ${ }^{\mathrm{a}}$

\begin{tabular}{lll}
\hline Inhibitor & \multicolumn{2}{l}{ Concentrations } \\
\hline Rapamycin & $20 \mathrm{nM}$ & $100 \mathrm{nM}$ \\
Everolimus & $20 \mathrm{nM}$ & $100 \mathrm{nM}$ \\
Deforolimus & $100 \mathrm{nM}$ & $1,000 \mathrm{nM}$ \\
Temsirolimous & $10 \mathrm{nM}$ & $100 \mathrm{nM}$ \\
Resveratrol & $25 \mu \mathrm{M}$ & $50 \mu \mathrm{M}$ \\
BEZ235 & $10 \mathrm{nM}$ & $100 \mathrm{nM}$
\end{tabular}

anhibitor concentrations were chosen to reflect the range that other studies had found effective.

ground measurement for each image was calculated by taking an average measurement from 5 different areas across the image. This average background measurement was subtracted from each of the images, producing the final data that were used for statistical analysis. This process was repeated for all 14 different images.

ImageStreamx Mark II flow cytometry. Whole blood (1 ml) from patient samples $(\mathrm{n}=3)$ was mixed with $9 \mathrm{ml}$ of red blood cell lysis (RBC) buffer (G Biosciences, St. Louis, MO, USA), followed by incubation for $10 \mathrm{~min}$ with gentle agitation. Following centrifugation at $1,260 \mathrm{x} \mathrm{g}$ for $10 \mathrm{~min}$ at $4^{\circ} \mathrm{C}$ the supernatant was discarded and $2 \mathrm{ml}$ of RBC lysis buffer was added. This was followed by incubation for $10 \mathrm{~min}$ at room temperature with gentle agitation. Another centrifugation took 


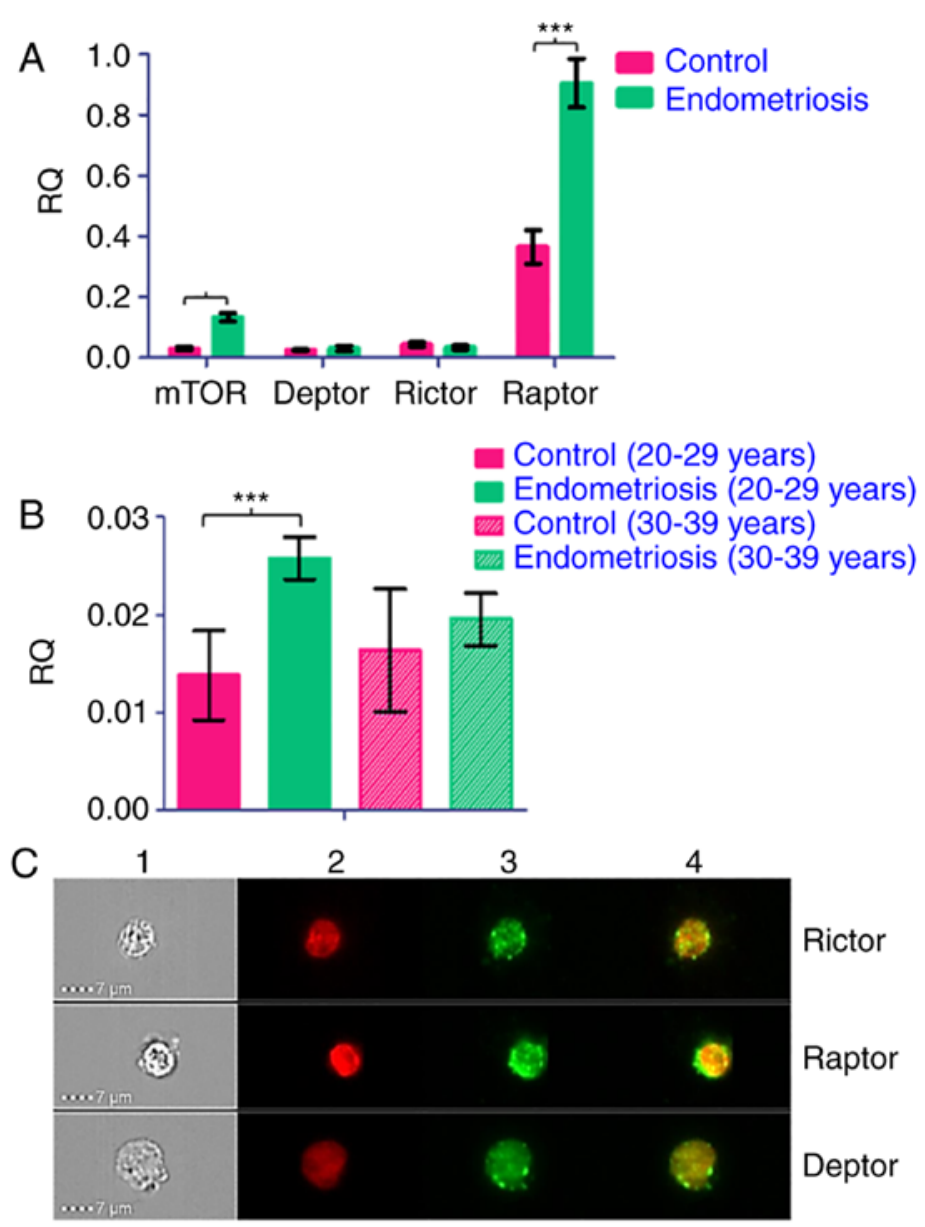

Figure 2. qPCR for mTOR, DEPTOR, Rictor and Raptor was carried out in triplicate. Error bars \pm SEM. mTOR and raptor showed a significant increase in expression compared to controls (A, $\mathrm{P} \leq 0.0001$ for both groups). Rictor showed a significant increase in patients aged 20-29 years ( $\mathrm{P}=0.0004)$ (B). Expression of Rictor, Raptor and DEPTOR in CTCs using ImageStreamx Mark II flow cytometry. Channel 1: Brightfield of CTCs; Channel 2: Staining of CTCs with the nuclear marker DRAQ5 (red); Channel 3: Expression of mTOR components (green); and Channel 4: Merged images from channels 2 and 3 (C). ${ }^{* * *} \mathrm{P}<0.001$.

place as previously described and the pellet was resuspended with $1.5 \mathrm{ml}$ of PBS. The solution containing CTCs (and some white blood cells) was centrifuged at $1,450 \mathrm{x} \mathrm{g}$ for $3 \mathrm{~min}$. The resulting cell pellet was resuspended in $1 \mathrm{ml}$ of ice-cold $4 \%$ paraformaldehyde for $7 \mathrm{~min}$ on ice and then centrifuged for $5 \mathrm{~min}$ at $250 \mathrm{x}$ g. Subsequent staining using specific antibodies against Rictor, Raptor and DEPTOR diluted in FBS-PBS (1:200) were used as previously described (24).

The qPCR data of the relative gene expression were analysed using the $\Delta \mathrm{Cq}$ method whereby the $\mathrm{Cq}$ value of the endogenous control was subtracted from the $\mathrm{Cq}$ value of the gene of interest to calculate the $\Delta \mathrm{Cq}$ and an RQ (relative quantity) value was calculated by finding $2^{-\Delta \Delta \mathrm{Cq}}(23)$. Where more than one reference gene was used, the RQ values were averaged.

Statistical analysis. Data were presented as means \pm SEM. All statistical tests were performed using Graph Pad Prism software. If homoscedasticity (variance) of data was proven, an unpaired, two-tailed Student's t-test was performed to assess significance in all cases as no matched pairs of samples were used. If data were not homoscedastic, an unpaired, two-tailed Student's t-test with Welch's correction was performed to account for variance. Survival information for TCGA ovarian samples $(n=426)$ was extracted from UCSC Xena browser repository (https://xenabrowser.net). Patient survival was measured in days to death or end-of-study. The samples were fractioned based on the mean gene expression value. The survival Kaplan-Meier curves were plotted in R using survfit function from bioconductor (https://bioconductor.org). $\mathrm{P}<0.05$ was considered statistically significant.

\section{Results}

Expression of mTOR, DEPTOR, Rictor and Raptor in endometriosis. qPCR for mTOR, DEPTOR, Rictor and Raptor was carried out using the reference genes RPL13A and YWHAZ in triplicate on cDNA synthesised from the extracted RNA from tissue of endometriosis patients and from non-affected controls. In endometriosis patients $m T O R$ and Raptor, but not DEPTOR, showed a significant increase in expression (Fig. 2A, $\mathrm{P} \leq 0.0001$ for both groups). Rictor showed no significant change overall but when segregated by age of patient (20-29 years and 30-39 years) and grade points $(<50$ and $>50)$ significant changes were evident. Rictor showed a significant increase in patients aged $20-29$ years $(\mathrm{P}=0.0004)$ but not in patients aged 30-39 years (Fig. 2B). We expanded our observations on the expression of mTOR components using liquid biopsies from ovarian cancer patients given that endometriosis is a risk factor for this malignancy. We observed that circulating tumour cells (CTCs) express Rictor, Raptor and DEPTOR (Fig. 2C). 

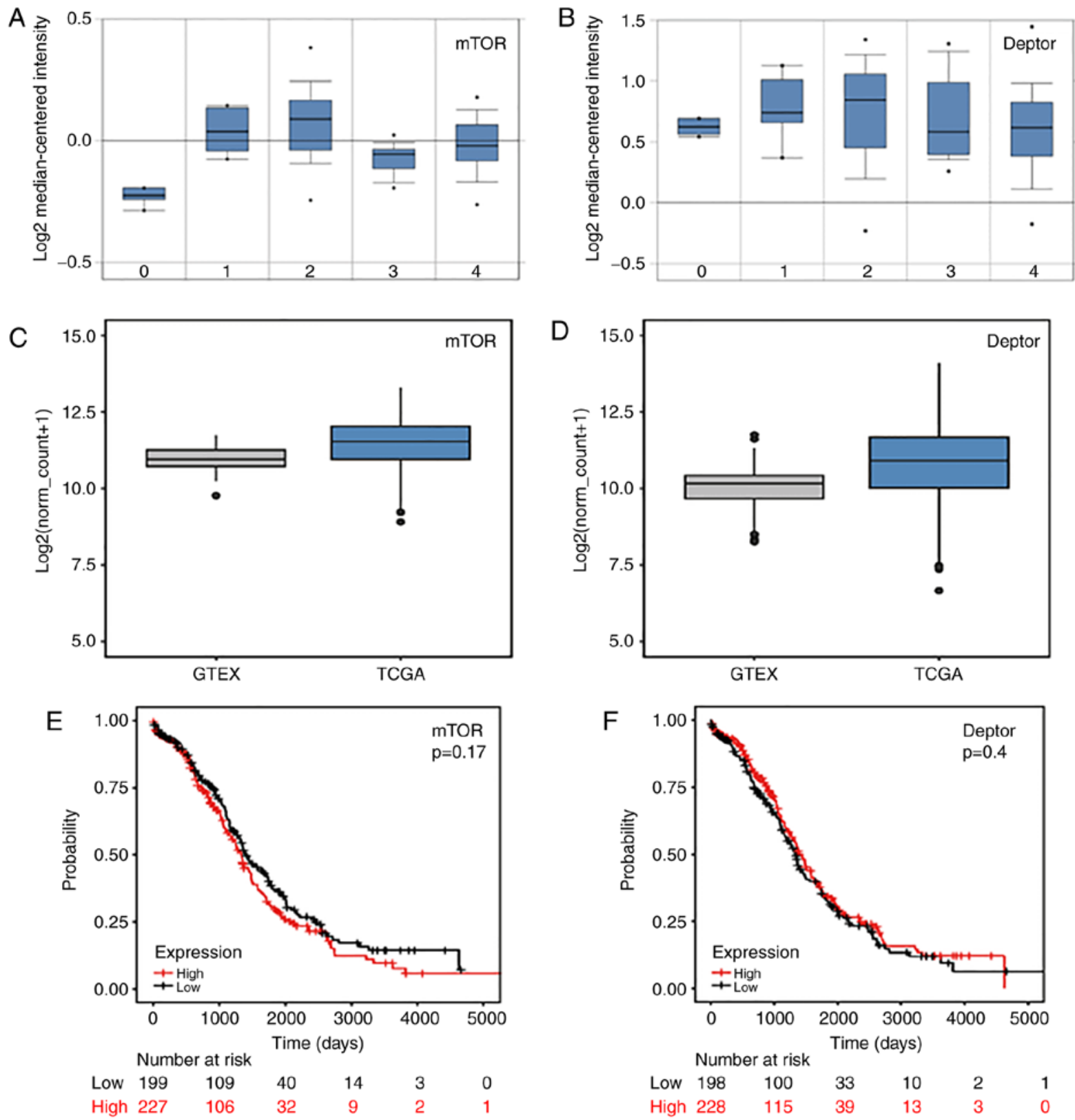

Figure 3. Expression levels of (A) mTOR and (B) DEPTOR in Hendrix dataset. 1) Control (n=4); 2) Ovarian Clear Cell Adenocarcinoma (n=8); 3) Ovarian Endometrioid Adenocarcinoma $(n=37) ; 4)$ Ovarian Mucinous Adenocarcinoma $(n=13), 5)$ Ovarian Serous Adenocarcinoma ( $\mathrm{n}=41)$. Boxplots showing the average expression level of the $m T O R(\mathrm{C})$ and DEPTOR $(\mathrm{D})$ genes in ovarian samples from TCGA serous cystadenocarcinoma and GTEX. The expression level unit is shown as a $\log 2$ transformed of the normalised count +1 , in order to facilitate cross-dataset comparison. Kaplan-Meier plots of overall survival (days) for higher and respectively lower than the mean expression level for mTOR (E) and DEPTOR (F).

Differential expression of $M T O R$ and DEPTOR in ovarian cancer. We analysed the expression of $m T O R$ and DEPTOR using the available RNAseq and microarray data. Specifically, we compared the expression of the two genes in various ovarian carcinomas using the Oncomine-curated dataset from Hendrix et al (25) (Fig. 2A and B). Both mTOR and DEPTOR showed an increase in the expression level compared to the control, across a variety of carcinomas, with mTOR being significantly upregulated in the ovarian endometrioid adenocarcinomas (Fig. 3A and B). In order to remove any potential bias induced by the small sample size of the Hendrix dataset $(n=103)$, we examined the gene expression in ovarian serous cystadenocarcinoma samples from TCGA $(n=428)$. As a control, we used the normal ovarian tissue samples from
GTEX ( $n=88)$. All the data were extracted from the UCSC Xena repository. Ovarian samples from TCGA and GTEX were mapped, processed, quantified, and normalised using the same pipeline as described in Vivian et al (26). As previously observed in the endometrioid dataset, both $m T O R$ and DEPTOR showed a statistically significant increase in the expression level in cancer samples compared to normal (p-val=5.26e-13 and 3.43e-13, respectively) (Fig. 3C and D). In terms of overall survival, we examined the Kaplan-Meier plots for the ovarian serous cystadenocarcinoma data from TCGA $(n=426)$. The samples were separated into high and low expression levels, respectively, based on the mean expression across the entire dataset (Fig. 3E and F). We observed that in the case of $m T O R$, a higher expression level is associated 
A
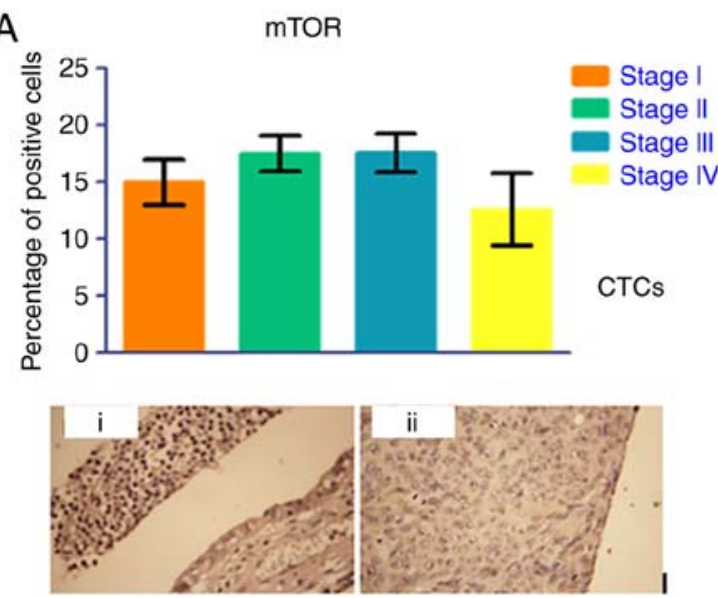

B
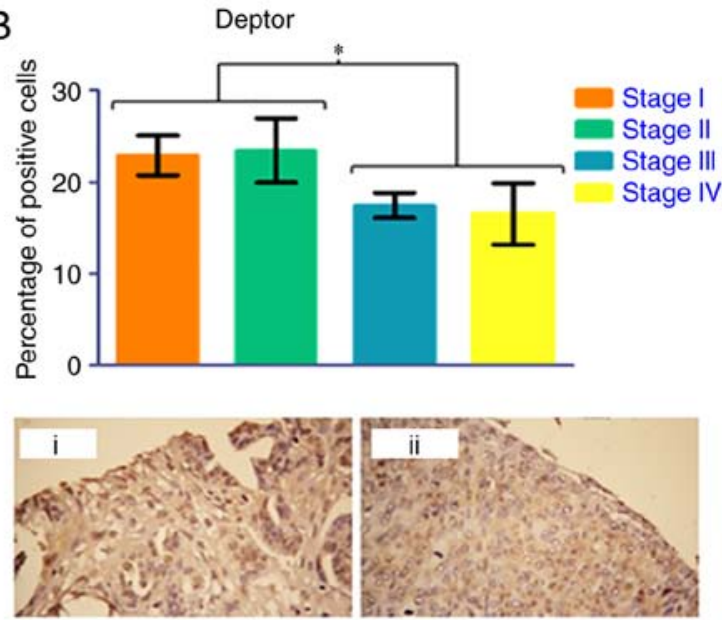

Figure 4. Immunohistochemistry for mTOR (A) and DEPTOR (B) was performed on paraffin-embedded ovarian tissue clinical samples from stage I-IV ovarian tumours. Error bars depict standard error. A significant difference was detected between early stage and late stage disease (P=0.0109). Panels Ai-Aii and Bi-Bii show representative images of stage I and stage IV serous papillary carcinoma respectively for mTOR and DEPTOR.

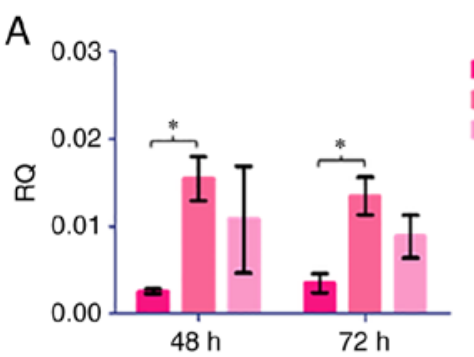

Deptor

Control

Rap $20 \mathrm{nM}$

Rap $100 \mathrm{nM}$
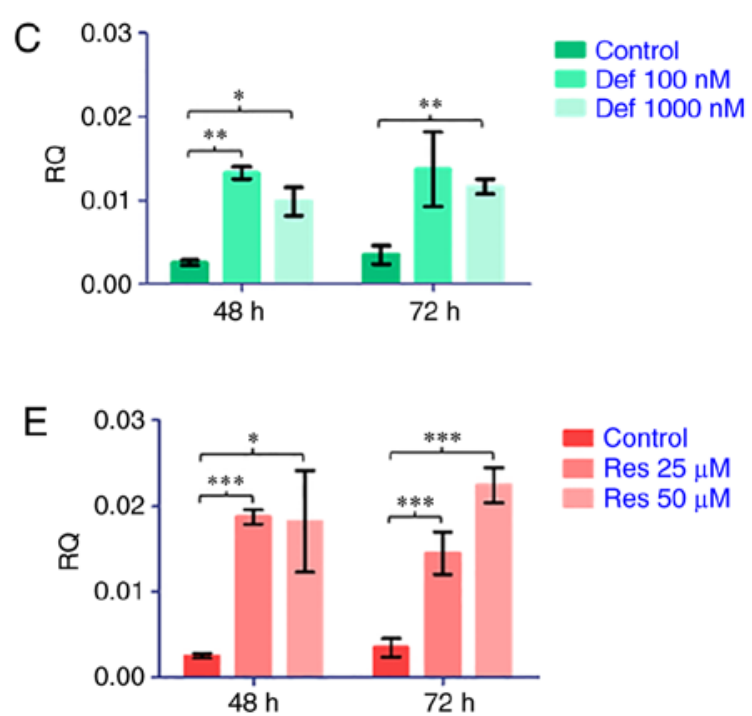

Figure 5. Relative DEPTOR expression was measured by qPCR in MDAH-2774 cells treated with Rap (A, 20 and $100 \mathrm{nM})$, Eve (B, 20 and $100 \mathrm{nM})$, Def $(\mathrm{C}, 100$ and $1,000 \mathrm{nM})$, Tem (D, $10 \mathrm{nM}$ and $100 \mathrm{nM}$ ), Res (E, 25 and $50 \mu \mathrm{M}), \mathrm{BEZ}(\mathrm{F}, 10$ and $100 \mathrm{nM})$ and carrier (DMSO) only control for 48 and $72 \mathrm{~h}$. cDNA was synthesised from extracted RNA from 3 biological replicates for each condition ${ }^{*} \mathrm{P}<0.05,{ }^{* * *} \mathrm{P}<0.01,{ }^{* * * *} \mathrm{P}<0.001$.

with a lower survival rate. By contrast, DEPTOR suggests that an increase in expression level is beneficial to the organisms' survival. However, this result is not statistically significant.

We expanded on these observations by assessing the protein expression of the two key components of mTORC1 and mTORC2 complexes using tissue microarray. mTOR expression at the protein level was identified throughout the stages
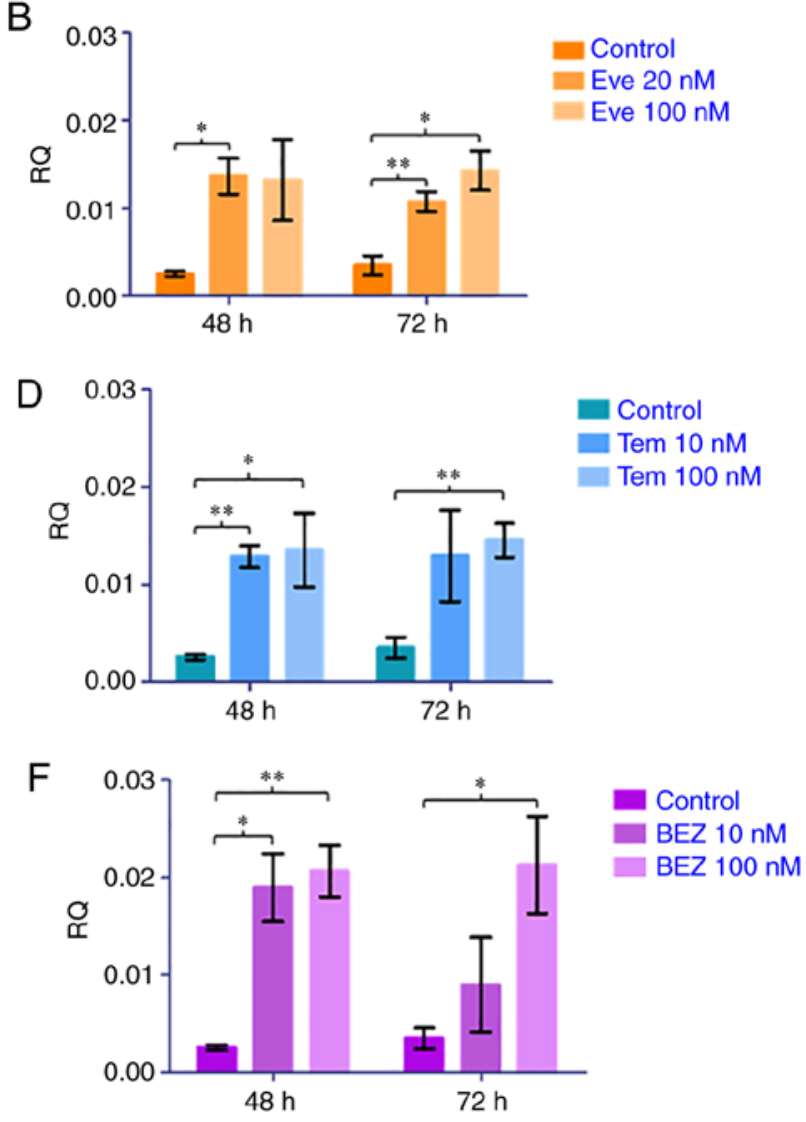
the other hand, showed a significant downregulation when we compared stages III and IV to stages I and II (Fig. 4A and B).

Effects of rapalogues on mTORC1 and mTORC2 components in vitro. Based on our clinical findings, it is evident that both $m T O R$ and DEPTOR are involved in ovarian cancer. For this 
Raptor
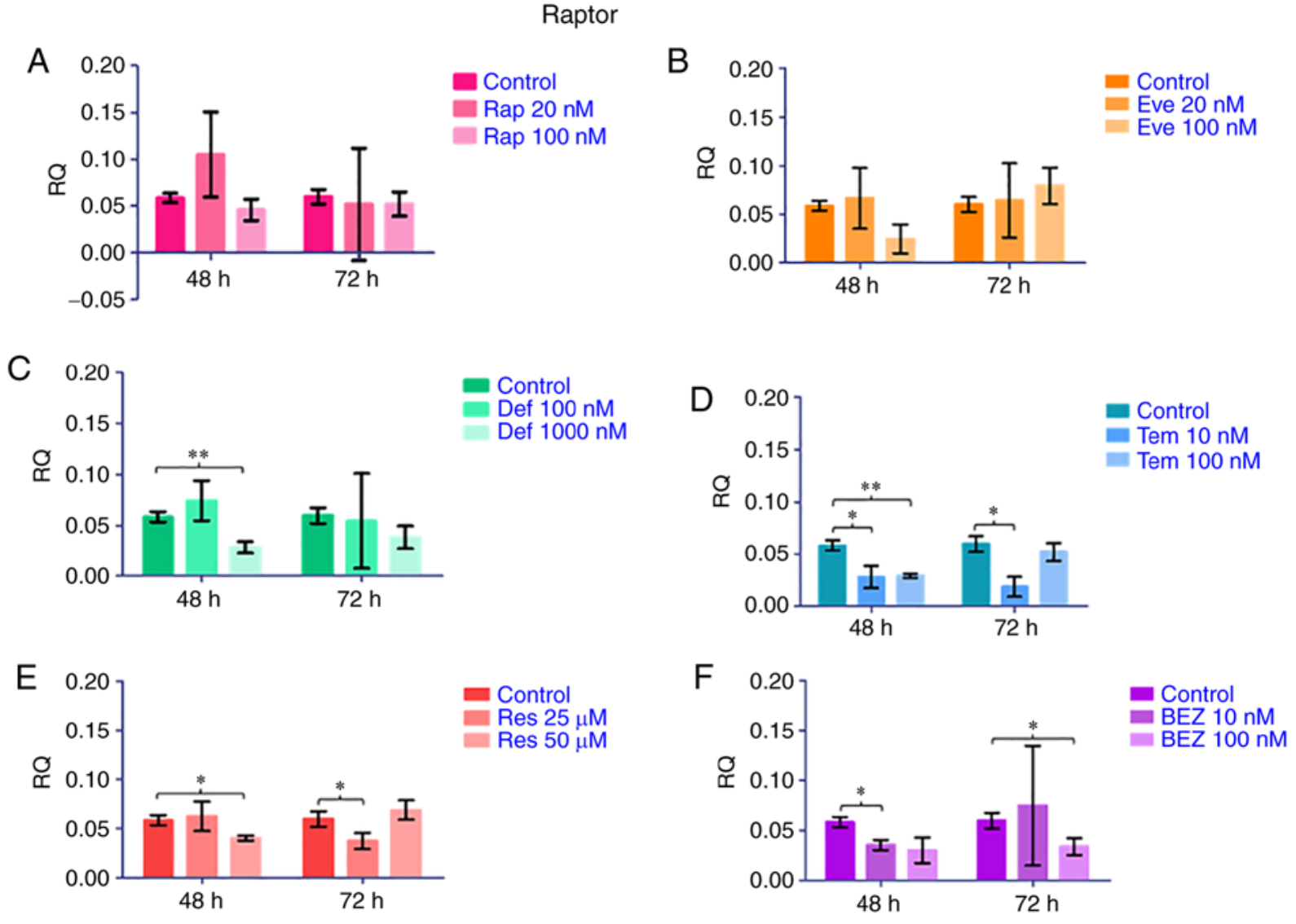

Figure 6. Relative Raptor expression was measured by qPCR in MDAH-2774 cells treated with Rap (A, 20 and $100 \mathrm{nM}$ ), Eve (B, 20 and $100 \mathrm{nM}$ ), Def (C, 100 and $1,000 \mathrm{nM}$ ), Tem (D, 10 and $100 \mathrm{nM}$ ), Res (E, 25 and $50 \mu \mathrm{M}), \mathrm{BEZ}\left(\mathrm{F}, 10\right.$ and $100 \mathrm{nM}$ ) and carrier (DMSO) only control for 48 and $72 \mathrm{~h} .{ }^{*} \mathrm{P}<0.05,{ }^{* * *} \mathrm{P}<0.01,{ }^{* * * *} \mathrm{P}<0.001$.

reason, we investigated the effects of rapalogues on mTORC1 and mTORC2 components in vitro, using the ovarian cancer cell line of endometrioid origin MDAH-2774 as an experimental model. MDAH-2774 cells were treated with Rapamycin, as well as the rapalogues Everolimus, Deforolimus, and Temsirolimus, NVP-BEZ235 and resveratrol. All the treatments led to a significant increase in the gene expression of DEPTOR (Fig. 5).

Subsequently, we assessed the expression of Raptor. MDAH-2774 cells showed an overall trend towards decreasing Raptor expression in response to mTOR pathway inhibition (Fig. 6). Rapamycin and Everolimus treatment induced no change in expression at any time point or in response to high or low concentrations in MDAH-2774 cells. Deforolimous treatment $(1,000 \mathrm{nM})$ induced a decrease in raptor expression after $48 \mathrm{~h}$ of treatment in comparison to the controls (Fig. 6C, $\mathrm{P}=0.0067)$. Temsirolimous treatment $(10 \mathrm{nM})$ induced a decrease in raptor expression after 48 and $72 \mathrm{~h}$ of treatment in MDAH-2774 cells in comparison to the controls (Fig. 6D, $\mathrm{P}=0.0457$ and 0.0101 , respectively). In addition, $100 \mathrm{nM}$ Tem treatment induced a decrease in Raptor expression after $48 \mathrm{~h}$ of treatment (Fig. 6D, $\mathrm{P}=0.0115)$. Resveratrol treatment $(25 \mu \mathrm{M})$ resulted in a decreased raptor expression after $72 \mathrm{~h}$ in comparison to controls and $50 \mu \mathrm{M}$ Res treatment had the same effect after 48-h treatment (Fig. 6E, $\mathrm{P}=0.0412$ and 0.0318 , respectively). BEZ treatment $(10 \mathrm{nM})$ induced a decrease in Raptor expression after $48 \mathrm{~h}$ of treatment and $100 \mathrm{nM}$ BEZ treatment resulted in decreased raptor expression after $72 \mathrm{~h}$ in comparison to controls (Fig. 6F, $\mathrm{P}=0.012$ and 0.0298 , respectively).
We also assessed the phosphorylation status of PRAS40 and the protein expression of G $\beta \mathrm{L}$ on MDAH-2774 cells treated with Rapamycin, Deforolimus, Resveratrol, Everolimus and Temsirolimus. Little change was observed at $48 \mathrm{~h}$; however, a reduction of phospho-PRAS40 following treatment with Rapamycin, Deforolimus, Everolimus and Temsirolimus was evident. Similarly, a slight decrease in G $\beta$ L following rapamycin treatment was also identified (data not shown).

Interestingly, although the same treatments did not significantly alter mTOR mRNA expression (data not shown), the dual kinase inhibitor NVP-BEZ235 as well rapamycin significantly decreased its protein expression at $48 \mathrm{~h}$ (Fig. 7). It is evident, therefore, that the differential expression of mTOR and DEPTOR is crucial for the activity of the complexes.

\section{Discussion}

In this study we provide evidence of differential expression of mTOR components in patients with endometriosis and ovarian cancer, and how rapalogues, including dual kinase inhibitors, can alter the expression of mTOR and DEPTOR. Collectively our data suggest a crucial role for DEPTOR in ovarian cancer. In patients with endometriosis there was a significant increase in the expression of mTOR and Raptor as compared to the controls. Rictor did not show a significant change in overall expression, indicating the involvement of mTORC1 rather than mTORC2. However, Rictor was significantly increased only in those aged 20-29 years. Proliferative 

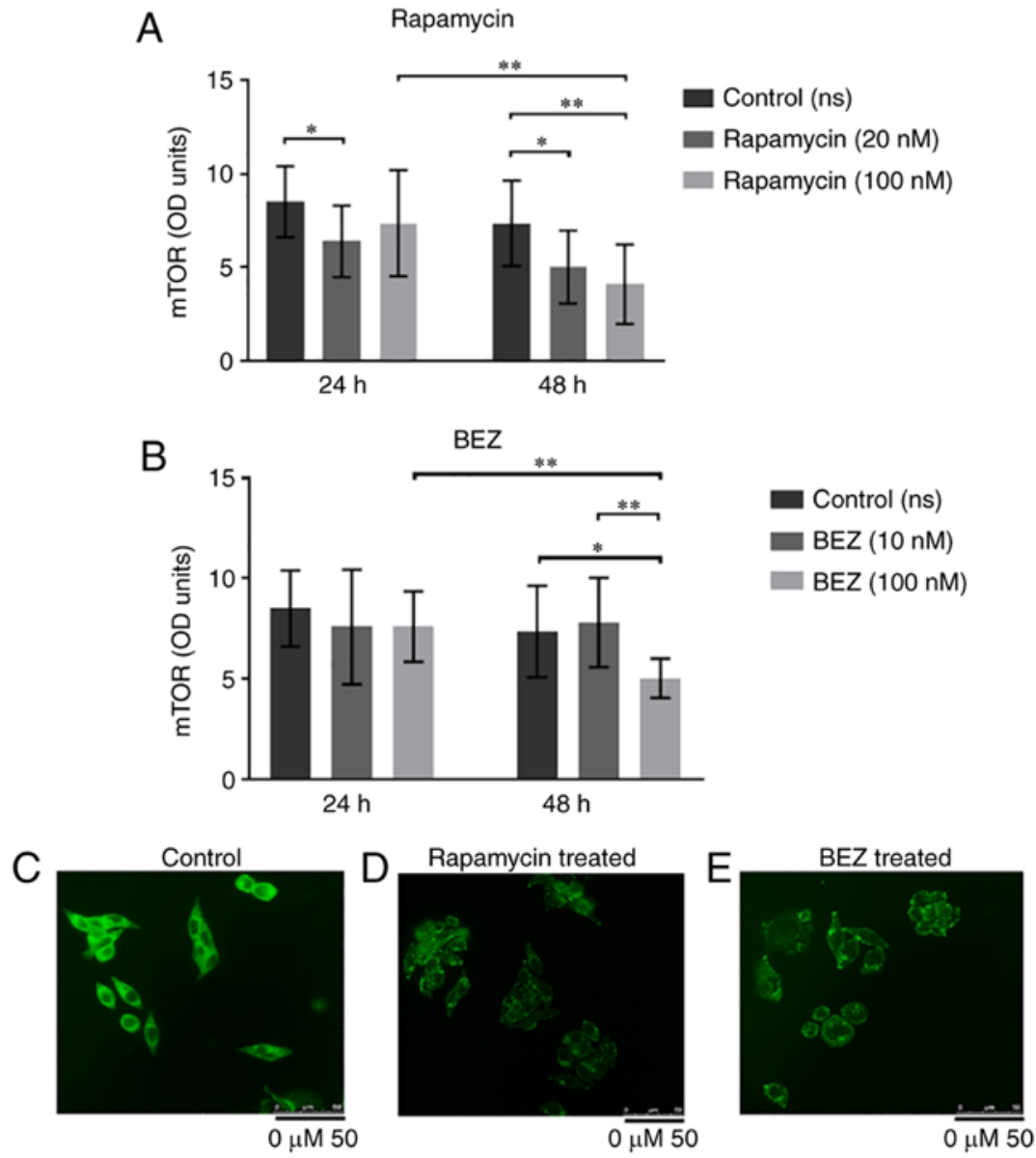

Figure 7. Effects of Rapamycin (A) and BEZ (B) on mTOR protein expression in MDAH-2774 cells. ${ }^{*} \mathrm{P}<0.05,{ }^{* *} \mathrm{P}<0.01$. Error bars \pm SEM. Panels $(\mathrm{C}-\mathrm{E})$ are photomicrographs of MDAH cells treated with rapamycin and BEZ compared to the control.

diseases of the endometrium such as endometriosis and cancer are estrogen-dependent. It is possible that steroids affect the expression and subsequent activity of mTOR pathway. In a recent study, it was shown that the activation of protein synthesis in mouse uterine epithelial cells by estradiol-17 $\beta$ is mediated via a PKC-ERK1/2-mTOR signalling pathway (27). In breast cancer, Rictor's expression was higher in estrogen receptor-positive cases than in estrogen receptor-negative cases. Similarly, there is a direct link between mTORC1 and $\mathrm{ER} \alpha$, since mTORC1 directly phosphorylates and activates ER $\alpha$ upon estrogen stimulation (28). In a large meta-analysis involving 444,255 patients from 1,625 relevant studies, endometriosis was strongly associated with the increased risk of ovarian cancer, and endometriosis-associated ovarian cancer (EAOC) showed favourable characteristics including early-stage disease, and a specific histology such as endometrioid or clear cell carcinoma (29).

The shift or change from endometriosis to ovarian cancer has been suggested to involve the mTOR pathway (30-35). Deletion of PTEN, a tumour suppressor gene upstream of mTOR and involved in the PI3 kinase/Akt/mTOR axis in mice, induced ovarian endometrioid adenocarcinoma. In addition, the combination of K-Ras phosphorylation and PTEN deletion induced the phosphorylation of Akt, mTOR and p70S6 kinase indicating that the mTOR pathway, either by PI3 kinase/Akt or Ras/MAP kinase activation is involved in the transformation of endometriosis to ovarian cancer (30).
This is further supported by the frequent downregulation of PTEN found in ovarian cancers $(31,32)$ and increased K-Ras activation in endometriosis $(33,34)$. In a more recent study using targeted next generation sequencing, it was shown that the PI3K-AKT-mTOR pathway may promote cell malignant transformation towards EAOC (35).

In the present study, a common pattern of expression was seen between ovarian cancer and endometriosis. We observed an increase in mTOR and Raptor expression in endometriosis, suggesting that mTOR complex 1 is involved in this condition as Raptor is an exclusive component of mTORC1. Previous findings have shown that, DEPTOR is involved in the pathogenesis of human malignancies, primarily via its inhibitory role towards mTORC1 and mTORC2 $(36,37)$. In addition, DEPTOR is underexpressed in many types of cancer including that of the prostate, bladder, and cervix, but it is overexpressed in multiple myelomas, thyroid cancers, and taxol-resistant ovarian cancer cell lines $(28,36,38)$.

In the present study, although we did not observe any marked changes in the expression of DEPTOR in either endometriosis or ovarian cancer patients, there was a significant decrease in its protein expression when we compared stages I and II to III and IV in ovarian cancer patients. This is consistent with the notion that deregulation of DEPTOR can contribute towards a 'hyperactive' mTOR pathway in cancer (39). DEPTOR can also be of prognostic value towards overall survival, since upregulation correlates positively with better 
prognosis. These data corroborate a previous study showing that DEPTOR expression is an independent prognostic marker for thyroid carcinoma (38). It has also been considered as a predictive biomarker for therapeutic response in MM patients treated with thalidomide (40). However, to date, the exact role of DEPTOR remains controversial due to its ability to function as an oncogene and as a tumour-suppressor gene, depending on its cellular or tissue distribution (40).

Our study opens prospects for the use of DEPTOR, Rictor, and Raptor as potential biomarkers. Over the past years, the concept of liquid biopsies has been introduced as an alternative to conventional tissue biopsy. Circulating tumour cells (CTCs) are cells that invade into the bloodstream from the primary tumours in the abdominal cavity $(41,42)$. These cells are different to normal circulating blood cells and express tumour-specific characteristics. CTCs are of diagnostic value in various types of cancer, but the clinical value of CTCs in ovarian cancer remains to be determined.

In order to gain better insight, we have used the MDAH-2774 endometrioid ovarian cancer cell line as a preclinical model to study the mTOR pathway in basal state and upon inhibition using a wide repertoire of rapalogues, resveratrol and a dual kinase inhibitor. The findings showed that, inhibition of the mTOR pathway causes a shift of the DEPTOR/mTOR gene ratio. Moreover, rapamycin and NVP-BEZ downregulated the protein expression of mTOR. We hypothesise that DEPTOR overexpression counteracts mTOR activity acting as a potential drug target. Previous studies have already demonstrated that use of rapalogues can be of therapeutic potential in ovarian cancer. Everolimus inhibited the proliferation of human ovarian cancer cells, and prolonged survival in an ovarian cancer mouse model in vivo (43). A more detailed analysis of mTORC1 and mTORC2 signalling must be carried out to fully explore the extent to which each pathway is active in endometriosis and ovarian cancer using appropriate in vivo models with a view to identify the most appropriate drug targets. Indeed, rapalogues, which robustly inhibit mTORC1 signalling but have little effect on mTORC2 signalling have not demonstrated any advances in cancer treatment that was expected from them, with few licenced uses for malignancy (14). Moreover, performing colocalization experiments on DEPTOR with mTORC1 and mTORC2 components under basal conditions and in the presence of mTOR inhibitors allows us to further understand the stoichiometry of DEPTOR within the mTOR complexes.

The present study is a proof-of-concept pilot study. This research can be extended in the future with the use of primary cell lines, pending ethical approval. Moreover, future studies would provide better insight into the role of mTOR inhibitors (including dual inhibitors and resveratrol) in the expression of key components such as Protor or mSIN1 at the gene or protein level. Moreover, to the best of our knowledge, we have mapped for the first time, the expression of DEPTOR, Rictor and Raptor in CTCs. Future studies should demonstrate whether their expression can be used as a novel biomarker for diagnostic or prognostic purposes.

Upregulation of DEPTOR is a positive prognostic marker in ovarian cancer and is increased in response to mTOR pathway inhibition suggesting that it functions as a tumour suppressor gene in endometrioid ovarian carcinoma. Collectively, our data suggest the mTOR pathway as a potential connection between endometriosis and ovarian cancer and may be a potential target in the treatment of both conditions (44).

\section{Acknowledgements}

Not applicable.

\section{Funding}

This study was funded by GRACE, Department of Gynaecological Oncology, The Royal Surrey County Hospital, Egerton Road, Guildford, Surrey GU2 7XX; and the Cancer Treatment and ResearchTrust (CTRT), Mount Vernon Hospital, Rickmansworth Road, Northwood, Middlesex, HA6 2RN, UK.

\section{Availability of data and materials}

All data generated or analyzed during this study are available from the corresponding author on reasonable request.

\section{Authors' contributions}

$\mathrm{EM}$ and $\mathrm{MH}$ contributed to the conception and design of the study. KRB, CS EM, JJ, EK and JK contributed to data acquisition and analysis. TG, GW, GP, DT, JC and EK were involved in data analysis and interpretation. TG, GW, JC and EK were involved in the drafting, writing and revision of the manuscript for intellectual content. All authors read and approved the final manuscript.

\section{Ethics approval and consent to participate}

Ethical approval was obtained by the Ethics Committee of Papageorgiou Hospital, Thessaloniki, Greece and the Research and Development department of East and North Hertfordshire NHS Trust, England, UK. Written informed consent was obtained from all the patients.

\section{Patient consent of publication}

Not applicable.

\section{Competing interests}

The authors declare that they have no competing interests.

\section{References}

1. Sinaii N, Cleary SD, Ballweg ML, Nieman LK and Stratton P: High rates of autoimmune and endocrine disorders, fibromyalgia, chronic fatigue syndrome and atopic diseases among women with endometriosis: A survey analysis. Hum Reprod 17: 2715-2724, 2002.

2. NHS Choices Endometriosis-NHS Choices. http://www.nhs. uk/conditions/endometriosis/pages/introduction.aspx. Accessed June 1, 2016.

3. Sainz de la Cuesta R, Eichhorn JH, Rice LW, Fuller AF Jr, Nikrui $\mathrm{N}$ and Goff BA: Histologic transformation of benign endometriosis to early epithelial ovarian cancer. Gynecol Oncol 60: 238-244, 1996.

4. Stewart LM, Holman CD, Aboagye-Sarfo P, Finn JC, Preen DB and Hart R: In vitro fertilization, endometriosis, nulliparity and ovarian cancer risk. Gynecol Oncol 128: 260-264, 2013. 
5. Pearce CL, Templeman C, Rossing MA, Lee A, Near AM, Webb PM, Nagle CM, Doherty JA, Cushing-Haugen KL, Wicklund KG, et al: Association between endometriosis and risk of histological subtypes of ovarian cancer: A pooled analysis of case-control studies. Lancet Oncol 13: 385-394, 2012.

6. Wu AH, Pearce CL, Tseng CC, Templeman C and Pike MC: Markers of inflammation and risk of ovarian cancer in Los Angeles County. Int J Cancer 124: 1409-1415, 2009.

7. Merritt MA, Green AC, Nagle CM and Webb PM; Australian Cancer Study (Ovarian Cancer); Australian Ovarian Cancer Study Group: Talcum powder, chronic pelvic inflammation and NSAIDs in relation to risk of epithelial ovarian cancer. Int J Cancer 122: 170-176, 2008

8. Melin A, Sparén P, Persson I and Bergqvist A: Endometriosis and the risk of cancer with special emphasis on ovarian cancer. Hum Reprod 21: 1237-1242, 2006.

9. Borgfeldt $\mathrm{C}$ and Andolf E: Cancer risk after hospital discharge diagnosis of benign ovarian cysts and endometriosis. Acta Obstet Gynecol Scand 83: 395-400, 2004.

10. Rossing MA, Cushing-Haugen KL, Wicklund KG, Doherty JA and Weiss NS: Risk of epithelial ovarian cancer in relation to benign ovarian conditions and ovarian surgery. Cancer Causes Control 19: 1357-1364, 2008.

11. Brinton LA, Gridley G, Persson I, Baron J and Bergqvist A Cancer risk after a hospital discharge diagnosis of endometriosis. Am J Obstet Gynecol 176: 572-579, 1997.

12. Brinton LA, Lamb EJ, Moghissi KS, Scoccia B, Althuis MD Mabie JE and Westhoff CL: Ovarian cancer risk associated with varying causes of infertility. Fertil Steril 82: 405-414, 2004.

13. Brinton LA, Sakoda LC, Sherman ME, Frederiksen K, Kjaer SK, Graubard BI, Olsen JH and Mellemkjaer L: Relationship of benign gynecologic diseases to subsequent risk of ovarian and uterine tumors. Cancer Epidemiol Biomarkers Prev 14: 2929-2935, 2005.

14. Laplante M and Sabatini DM: mTOR signaling in growth control and disease. Cell 149: 274-293, 2012.

15. Zoncu R, Efeyan A and Sabatini DM: mTOR: From growth signal integration to cancer, diabetes and ageing. Nat Rev Mol Cell Biol 12: 21-35, 2011.

16. Rogers-Broadway KR, Chudasama D, Pados G, Tsolakidis D, Goumenou A, Hall M and Karteris E: Differential effects of rapalogues, dual kinase inhibitors on human ovarian carcinoma cells in vitro. Int J Oncol 49: 133-143, 2016.

17. Leconte M, Nicco C, Ngô C, Chéreau C, Chouzenoux S, Marut W, Guibourdenche J, Arkwright S, Weill B, Chapron C, et al: The mTOR/AKT inhibitor temsirolimus prevents deep infiltrating endometriosis in mice. Am J Pathol 179: 880-889, 2011.

18. Afshar Y, Hastings J, Roqueiro D, Jeong JW, Giudice LC and Fazleabas AT: Changes in eutopic endometrial gene expression during the progression of experimental endometriosis in the baboon, Papio anubis. Biol Reprod 88: 44, 2013

19. Potter CJ, Pedraza LG and Xu T: Akt regulates growth by directly phosphorylating Tsc2. Nat Cell Biol 4: 658-665, 2002.

20. Manning BD, Tee AR, Logsdon MN, Blenis J and Cantley LC: Identification of the tuberous sclerosis complex-2 tumor suppressor gene product tuberin as a target of the phosphoinositide 3-kinase/akt pathway. Mol Cell 10: 151-162, 2002.

21. Ma XM and Blenis J: Molecular mechanisms of mTOR-mediated translational control. Nat Rev Mol Cell Biol 10: 307-318, 2009.

22. Guo J, Gao J, Yu X, Luo H, Xiong X and Huang O: Expression of DJ-1 and mTOR in eutopic and ectopic endometria of patients with endometriosis and adenomyosis. Gynecol Obstet Invest 79: 195-200, 2015

23. Foster H, Coley HM, Goumenou A, Pados G, Harvey A and Karteris E: Differential expression of mTOR signalling components in drug resistance in ovarian cancer. Anticancer Res 30: 3529-3534, 2010.

24. Chudasama D, Bo V, Hall M, Anikin V, Jeyaneethi J, Gregory J, Pados G, Tucker A, Harvey A, Pink R and Karteris E: Identification of cancer biomarkers of prognostic value using specific gene regulatory networks (GRN): A novel role of RAD51AP1 for ovarian and lung cancers. Carcinogenesis 39: 407-417, 2018

25. Hendrix ND, Wu R, Kuick R, Schwartz DR, Fearon ER and Cho KR: Fibroblast growth factor 9 has oncogenic activity and is a downstream target of Wnt signaling in ovarian endometrioid adenocarcinomas. Cancer Res 66: 1354-1362, 2006

26. Vivian J, Rao AA, Nothaft FA, Ketchum C, Armstrong J, Novak A, Pfeil J, Narkizian J, Deran AD, Musselman-Brown A, et al: Toil enables reproducible, open source, big biomedical data analyses. Nat Biotechnol 35: 314-316, 2017.
27. Wang Y, Zhu L, Kuokkanen S and Pollard JW: Activation of protein synthesis in mouse uterine epithelial cells by estradiol-17 $\beta$ is mediated by a PKC-ERK1/2-mTOR signaling pathway. Proc Natl Acad Sci USA 112: 1382-1391, 2015.

28. Alayev A, Salamon RS, Berger SM, Schwartz NS, Cuesta R, Snyder RB and Holz MK: mTORC1 directly phosphorylates and activates ER $\alpha$ upon estrogen stimulation. Oncogene 35: 3535-3543, 2016.

29. Kim HS, Kim TH, Chung HH and Song YS: Risk and prognosis of ovarian cancer in women with endometriosis: A meta-analysis. Br J Cancer 110: 1878-1890, 2014.

30. Dinulescu DM, Ince TA, Quade BJ, Shafer SA, Crowley D and Jacks T: Role of K-ras and Pten in the development of mouse models of endometriosis and endometrioid ovarian cancer. Nat Med 11: 63-70, 2005.

31. Laudański P, Kowalczuk O, Klasa-Mazurkiewicz D, Milczek T, Rysak-Luberowicz D, Garbowicz M, Baranowski W, Charkiewicz R, Szamatowicz J and Chyczewski L: Selective gene expression profiling of mTOR-associated tumor suppressor and oncogenes in ovarian cancer. Folia Histochem Cytobiol 49: 317-324, 2011.

32. De Marco C, Rinaldo N, Bruni P, Malzoni C, Zullo F, Fabiani F, Losito S, Scrima M, Marino FZ, Franco R, et al: Multiple genetic alterations within the PI3K pathway are responsible for AKT activation in patients with ovarian carcinoma. PLoS One 8: e55362, 2013

33. Shahrabi-Farahani M, Shahbazi S, Mahdian R and Amini-Moghaddam S: K-Ras 4A Transcript variant is up-regulated in eutopic endometrium of endometriosis patients during proliferative phase of menstrual cycle. Arch Gynecol Obstet 292: 225-229, 2015.

34. Amemiyaa S, Sekizawaa A, Otsukaa J, Tachikawa T, Saito H and Okai T: Malignant transformation of endometriosis and genetic alterations of K-ras and microsatellite instability. Int J Gynaecol Obstet 86: 371-376, 2004.

35. Er TK, Su YF, Wu CC, Chen CC, Wang J, Hsieh TH, Herreros-Villanueva M, ChenWT, Chen YT, Liu TC, et al: Targeted next-generation sequencing for molecular diagnosis of endometriosis-associated ovarian cancer. J Mol Med (Berl) 94: 835-847, 2016.

36. Peterson TR, Laplante M, Thoreen CC, Sancak Y, Kang SA, Kuehl WM, Gray NS and Sabatini DM: DEPTOR is an mTOR inhibitor frequently overexpressed in multiple myeloma cells and required for their survival. Cell 137: 873-886, 2009.

37. Kazi AA, Hong-Brown L, Lang SM and Lang CH: Deptor knockdown enhances mTOR Activity and protein synthesis in myocytes and ameliorates disuse muscle atrophy. Mol Med 17: 925-936, 2011.

38. Pei L, Xie P, Zhou E, Yang Q, Luo Y and Tang Z: Overexpression of DEP domain containing mTOR-interacting protein correlates with poor prognosis in differentiated thyroid carcinoma. Mol Med Rep 4: 817-823, 2011.

39. Wang Z, Zhong J, Inuzuka H, Gao D, Shaik S, Sarkar FH and Wei W: An evolving role for DEPTOR in tumor development and progression. Neoplasia 14: 368-375, 2012.

40. Catena V and Fanciulli M: Deptor: Not only a mTOR inhibitor. J Exp Clin Cancer Res 36: 12, 2017

41. Chudasama DY, Freydina DV, Freidin MB, Leung M, Montero Fernandez A, Rice A, Nicholson AG, Karteris E, Anikin V and Lim E: Inertia based microfluidic capture and characterisation of circulating tumour cells for the diagnosis of lung cancer. Ann Transl Med 4: 480, 2016.

42. Chudasama D, Burnside N, Beeson J, Karteris E, Rice A and Anikin V: Perioperative detection of circulating tumour cells in patients with lung cancer. Oncol Lett 14: 1281-1286, 2017.

43. Mabuchi S, Altomare DA, Cheung M, Zhang L, Poulikakos PI, Hensley HH, Schilder RJ, Ozols RF and Testa JR: RAD001 inhibits human ovarian cancer cell proliferation, enhances cisplatin-induced apoptosis, and prolongs survival in an ovarian cancer model. Clin Cancer Res 13: 4261-4270, 2007.

44. Grunt TW and Mariani GL: Novel approaches for molecular targeted therapy of breast cancer: Interfering with PI3K/AKT/mTOR signaling. Curr Cancer Drug Targets 13: 188-204, 2013

This work is licensed under a Creative Commons Attribution-NonCommercial-NoDerivatives 4.0 International (CC BY-NC-ND 4.0) License. 